\title{
Congruence successions in compositions
}

\author{
Toufik Mansour I" $\quad$ Mark Shattuck $\|^{*} \quad$ Mark C. Wilson非 \\ ${ }^{1}$ Department of Mathematics, University of Haifa, 3498838 Haifa, Israel \\ ${ }^{2}$ Department of Mathematics, University of Tennessee, Knoxville, TN 37996 USA \\ ${ }^{3}$ Department of Computer Science, University of Auckland, Private Bag 92019 Auckland, New Zealand \\ received $13^{\text {th }}$ July 2013, revised $20^{\text {th }}$ Jan. 2014, accepted $12^{\text {th }}$ May 2014.
}

\begin{abstract}
A composition is a sequence of positive integers, called parts, having a fixed sum. By an $m$-congruence succession, we will mean a pair of adjacent parts $x$ and $y$ within a composition such that $x \equiv y(\bmod m)$. Here, we consider the problem of counting the compositions of size $n$ according to the number of $m$-congruence successions, extending recent results concerning successions on subsets and permutations. A general formula is obtained, which reduces in the limiting case to the known generating function formula for the number of Carlitz compositions. Special attention is paid to the case $m=2$, where further enumerative results may be obtained by means of combinatorial arguments. Finally, an asymptotic estimate is provided for the number of compositions of size $n$ having no $m$-congruence successions.
\end{abstract}

Keywords: composition, parity succession, combinatorial proof, asymptotic estimate

\section{Introduction}

Let $n$ be a positive integer. A composition $\sigma=\sigma_{1} \sigma_{2} \cdots \sigma_{d}$ of $n$ is any sequence of positive integers whose sum is $n$. Each summand $\sigma_{i}$ is called a part of the composition. If $n, d \geq 1$, then let $\mathcal{C}_{n, d}$ denote the set of compositions of $n$ having exactly $d$ parts and $\mathcal{C}_{n}=\cup_{d=1}^{n} \mathcal{C}_{n, d}$. By convention, there is a single composition of $n=0$ having zero parts.

If $m \geq 1$ and $0 \leq r \leq m-1$, by an (m,r)-congruence succession within a sequence $\pi=\pi_{1} \pi_{2} \cdots \pi_{d}$, we will mean an index $i$ for which $\pi_{i+1} \equiv \pi_{i}+r(\bmod m)$. An $(m, r)$-congruence succession in which $r=0$ will be referred to as an $m$-congruence succession, the $m=2$ case being termed a parity succession (or just a succession). A sequence is said to be parity-alternating if it contains no parity successions, that is, if its terms alternate between even and odd values. This concept of parity succession for sequences extends an earlier one that was introduced for subsets [15] and later considered on permutations [16]. The terminology is an adaptation of an analogous usage in which a succession refers to a pair $p_{i}, p_{i+1}$ within an integral sequence $p_{1} p_{2} \cdots$ such that $p_{i+1}=p_{i}+1$ (see, e.g., [1, 21, 12]). For other related

\footnotetext{
*Email: tmansoureuniv.haifa.ac.il.

†Email: shattuck@math.utk.edu.

‡Email: mcw@es. auckland.ac.nz.
} 
problems involving restrictions on compositions, the reader is referred to the text [11] and such papers as [2, 3, 4, 5, 7, 10].

Enumerating finite discrete structures according to the parity of individual elements perhaps started with the formula of Tanny [22] for the number $g(n, k)$ of alternating $k$-subsets of $[n]$ given by

$$
g(n, k)=\left(\begin{array}{c}
\left\lfloor\frac{n+k}{2}\right\rfloor \\
k
\end{array}\right)+\left(\begin{array}{c}
\left\lfloor\frac{n+k-1}{2}\right\rfloor \\
k
\end{array}\right), \quad 1 \leq k \leq n .
$$

This result was recently generalized to any modulus in [13] and in terms of counting by successions in [15]. Tanimoto [20] considered a comparable version of the problem on permutations in his investigation of signed Eulerian numbers. There one finds the formula for the number $h(n)$ of parity-alternating permutations of length $n$ given by

$$
h(n)=\frac{3+(-1)^{n}}{2}\left\lfloor\frac{n}{2}\right\rfloor !\left\lfloor\frac{n+1}{2}\right\rfloor !,
$$

which has been generalized in terms of succession counting in [16]; see also [14].

In the next section, we consider the problem of counting compositions of $n$ according to the number of $(m, r)$-congruence successions, as defined above, and derive an explicit formula for the generating function for all $m$ and $r$ (see Theorem 2 below). When $r=0$, we obtain as a corollary a relatively simple expression for the generating function $F_{m}$ which counts compositions according to the number of $m$-congruence successions. Letting $m \rightarrow \infty$ and taking the variable in $F_{m}$ which marks the number of $m$-congruence successions to be zero recovers the generating function for the number of Carlitz compositions, i.e., those having no consecutive parts equal; see, e.g., [9].

In the third section, we obtain some enumerative results concerning the case $m=2$. In particular, we provide a bijective proof for a related recurrence and enumerate, in two different ways, the parityalternating compositions of size $n$. As a consequence, we obtain a combinatorial proof of a pair of binomial identities which we were unable to find in the literature. In the final section, we provide asymptotic estimates for the number of compositions of $n$ having no $m$-congruence successions as $n \rightarrow \infty$, which may be extended to compositions having any fixed number of successions.

Special cases of the generating function $R_{m, r}(x, y, q)$ defined below do fall within the general framework of Bender and Canfield in [2, 3, 4, 5]. In particular, if one takes $\mathcal{D}=\mathbb{Z}-\{k m+r: k \in \mathbb{Z}\}$ in Theorem 4 of [2], then one obtains another recurrence satisfied by the $q=0$ case of $R_{m, r}(x, y, q)$. We are unable to solve this recurrence explicitly in our case and no general solution to the recurrence in this theorem can apparently be found, as remarked by the authors. We note that the set $\mathcal{D}$ above was not among the examples considered in [2] or in the later paper [4]. Furthermore, the distribution of the statistic recording the number of adjacent differences belonging to a certain residue class mod $m$ has apparently not been previously studied.

\section{Counting compositions by number of $(m, r)$-congruence suc- cessions}

Let $c l_{m, r}(\pi)$ denote the number of $(m, r)$-congruence successions within a sequence $\pi=\pi_{1} \pi_{2} \cdots \pi_{d}$. Let $R_{m, r ; a}(x, y, q)=R_{a}(x, y, q)$ be the generating function for the number of compositions of $n$ with 
exactly $d$ parts whose first part is $a$ according to the statistic $c l_{m, r}$, that is,

$$
R_{a}(x, y, q)=\sum_{n \geq 0} \sum_{d=0}^{n} x^{n} y^{d}\left(\sum_{\pi=a \pi^{\prime} \in \mathcal{C}_{n, d}} q^{c l_{m, r}(\pi)}\right) .
$$

Clearly, we have $R_{m+a}(x, y, q)=x^{m} R_{a}(x, y, q)$ for all $a \geq 1$. Let $R_{m, r}(x, y, q)=R(x, y, q)=$ $1+\sum_{a \geq 1} R_{a}(x, y, q)$. By the definitions, we have

$$
R_{a}(x, y, q)=x^{a} y R(x, y, q)+x^{a} y(q-1) \sum R_{t}(x, y, q)
$$

for all $a \geq 1$, where the sum is taken over all positive integers $t$ such that $t \equiv a+r(\bmod m)$. Hence,

$$
\sum_{i \geq 0} R_{i m+a}(x, y, q)=\frac{x^{a} y}{1-x^{m}} R(x, y, q)+\frac{x^{a} y(q-1)}{1-x^{m}} \sum_{i \geq 0} R_{i m+a+r}(x, y, q),
$$

if $1 \leq a \leq m-r$, and

$$
\sum_{i \geq 0} R_{i m+a}(x, y, q)=\frac{x^{a} y}{1-x^{m}} R(x, y, q)+\frac{x^{a} y(q-1)}{1-x^{m}} \sum_{i \geq 0} R_{i m+a+r-m}(x, y, q),
$$

if $m-r+1 \leq a \leq m$. The last two equalities may be expressed as

$$
\begin{aligned}
& G_{j}(x, y, q)=\frac{x^{j} y}{1-x^{m}} R(x, y, q)+\frac{x^{j} y(q-1)}{1-x^{m}} G_{j+r}(x, y, q), \quad 1 \leq j \leq m-r, \\
& G_{j}(x, y, q)=\frac{x^{j} y}{1-x^{m}} R(x, y, q)+\frac{x^{j} y(q-1)}{1-x^{m}} G_{j+r-m}(x, y, q), \quad m-r+1 \leq j \leq m,
\end{aligned}
$$

where $G_{j}(x, y, q)=\sum_{i>0} R_{i m+j}(x, y, q)$.

In order to find an explicit formula for $G_{j}(x, y, q)$, we will need the following lemma.

Lemma 1. Suppose $x_{j}=a_{j}+b_{j} x_{j+r}$ for all $j=1,2, \ldots, m-r$ and $x_{j}=a_{j}+b_{j} x_{j+r-m}$ for all $j=m-r+1, m-r+2, \ldots, m$. Let $s=\operatorname{gcd}(m, r)$ and $p=m / s$. Then for all $j=1,2, \ldots, s$ and $\ell=0,1, \ldots, p-1$, we have

$$
x_{j+\ell r}=\sum_{i=\ell}^{\ell+p-1} \frac{a_{j+i r} \prod_{k=\ell}^{i-1} b_{j+k r}}{1-\prod_{k=\ell}^{\ell+p-1} b_{j+k r}},
$$

where $x_{j+m}=x_{j}, a_{j+m}=a_{j}$ and $b_{j+m}=b_{j}$.

Proof: Let $j=1,2, \ldots, s$. By definition of the sequence $x_{j}$ and $m$-periodicity, we may write

$$
\begin{aligned}
x_{j} & =a_{j}+b_{j} x_{j+r}=a_{j}+b_{j} a_{j+r}+b_{j} b_{j+r} x_{j+2 r} \\
& =\cdots=a_{j}+b_{j} a_{j+r}+\cdots+b_{j} b_{j+r} \cdots b_{j+(p-2) r} a_{j+(p-1) r}+b_{j} b_{j+r} \cdots b_{j+(p-1) r} x_{j+p r} .
\end{aligned}
$$

Since $p r \equiv 0(\bmod m)$, we have

$$
x_{j}=\sum_{i=0}^{p-1} \frac{a_{j+i r} \prod_{k=0}^{i-1} b_{j+k r}}{1-\prod_{k=0}^{p-1} b_{j+k r}} .
$$


More generally, for any $\ell=0,1, \ldots, p-1$,

$$
x_{j+\ell r}=\sum_{i=\ell}^{\ell+p-1} \frac{a_{j+i r} \prod_{k=\ell}^{i-1} b_{j+k r}}{1-\prod_{k=\ell}^{\ell+p-1} b_{j+k r}} .
$$

Let us denote by $\bar{t}$ the member of $\{1,2, \ldots, m\}$ such that $t \equiv \bar{t}(\bmod m)$ for a positive integer $t$. When $a_{j}=\frac{x^{j} y}{1-x^{m}} R(x, y, q)$ and $b_{j}=\frac{x^{j} y(q-1)}{1-x^{m}}$ for $1 \leq j \leq m$ in Lemma 1 , we get

$$
\begin{aligned}
x_{j+\ell r} & =\sum_{i=\ell}^{\ell+p-1} \frac{\frac{x^{\overline{j+i r}} y}{1-x^{m}} R(x, y, q) \prod_{k=\ell}^{i-1} \frac{x^{\overline{j+k r}} y(q-1)}{1-x^{m}}}{1-\prod_{k=\ell}^{\ell+p-1} \frac{x^{\overline{j+k r}} y(q-1)}{1-x^{m}}} \\
& =\frac{R(x, y, q)}{1-\left(\frac{y(q-1)}{1-x^{m}}\right)^{p} \prod_{k=\ell}^{\ell+p-1} x^{\overline{j+k r}}} \sum_{i=0}^{p-1} \frac{x^{\overline{j+(i+\ell) r}} y^{i+1}(q-1)^{i} \prod_{k=\ell}^{i+\ell-1} x^{\overline{j+k r}}}{\left(1-x^{m}\right)^{i+1}}
\end{aligned}
$$

for all $j=1,2, \ldots, s$ and $\ell=0,1, \ldots, p-1$, where $s=\operatorname{gcd}(m, r)$ and $p=m / s$. By (1), we have $G_{\overline{j+\ell r}}(x, y, q)=x_{j+\ell r}=x_{\overline{j+\ell r}}$, where $x_{j+\ell r}$ is given by (2). Note that the set of indices $j+\ell r$ for $1 \leq j \leq s$ and $0 \leq \ell \leq p-1$ is a complete residue set $(\bmod m)$. Using (2) and the fact that $R(x, y, q)=1+\sum_{a=1}^{m} G_{a}(x, y, q)$, we obtain the following result.

Theorem 2. If $m \geq 1,0 \leq r \leq m-1, s=\operatorname{gcd}(m, r)$ and $p=m / s$, then

$$
R_{m, r}(x, y, q)=\frac{1}{1-\sum_{j=1}^{s} \sum_{\ell=0}^{p-1} \sum_{i=0}^{p-1} \frac{x^{\overline{j+(i+\ell) r}} y^{i+1}(q-1)^{i} \prod_{k \ell}^{i+\ell-1} x^{\overline{j+k r}}}{\left(1-x^{m}\right)^{i+1}\left(1-\left(\frac{y(q-1)}{1-x^{m}}\right)^{p} \prod_{k=\ell}^{\ell+p-1} x^{\overline{j+k r}}\right)}}
$$

Note that in general we are unable to simplify the number theoretic product $\prod_{k=\ell}^{i+\ell-1} x^{\overline{j+k r}}$ appearing in (3).

Let

$$
F_{m}(x, y, q)=\sum_{n \geq 0} \sum_{d=0}^{n} x^{n} y^{d}\left(\sum_{\pi \in \mathcal{C}_{n, d}} q^{c l_{m}(\pi)}\right),
$$

where $c l_{m}(\pi)$ denotes the number of $m$-congruence successions of a sequence $\pi$. Taking $r=0$ in (3), and noting $s=\operatorname{gcd}(m, 0)=m$, gives the following result.

Corollary 3. If $m \geq 1$, then

$$
F_{m}(x, y, q)=\frac{1}{1-\sum_{a=1}^{m}\left(\frac{x^{a} y}{1-x^{m}-x^{a} y(q-1)}\right)} .
$$

Letting $q=0$ and $m \rightarrow \infty$ in (4) yields the generating function for the number of compositions having no $m$-congruence successions for all large $m$. Note that the only possible such compositions are those having no two adjacent parts the same. Thus, we get the following formula for the generating function which counts the Carlitz compositions according to the number of parts. 
Corollary 4. We have

$$
F_{\infty}(x, y, 0)=\frac{1}{1-\sum_{a=1}^{\infty} \frac{x^{a} y}{1+x^{a} y}}
$$

Let us close this section with a few remarks.

Remark 1. Letting $q=1$ in (3) gives

$$
R_{m, r}(x, y, 1)=\frac{1}{1-\frac{y}{1-x^{m}} \sum_{j=1}^{s} \sum_{\ell=0}^{p-1} x^{\overline{j+\ell r}}}=\frac{1}{1-\frac{y}{1-x^{m}} \sum_{a=1}^{m} x^{a}}=\frac{1-x}{1-x-x y}
$$

which agrees with the generating function for compositions where $x$ marks the size and $y$ marks the number of parts.

Remark 2. In [6], the generating function for the number $c(n, d)$ of Carlitz compositions of $n$ having $d$ parts was obtained as

$$
\sum_{n \geq 0} \sum_{d=0}^{n} c(n, d) x^{n} y^{d}=\frac{1}{1+\sum_{j \geq 1} \frac{(-x y)^{j}}{1-x^{j}}}
$$

Note that formulas (5) and (6) are seen to be equivalent since

$$
\sum_{a \geq 1} \frac{x^{a} y}{1+x^{a} y}=\sum_{a \geq 1} \sum_{j \geq 1}(-1)^{j-1} x^{a j} y^{j}=\sum_{j \geq 1}(-1)^{j-1} y^{j} \sum_{a \geq 1} x^{j a}=\sum_{j \geq 1}(-1)^{j-1} \frac{(x y)^{j}}{1-x^{j}}
$$

Remark 3. Letting $m=1$ in (4) gives

$$
F_{1}(x, y, q)=\frac{1-x-x y(q-1)}{1-x-x y q}
$$

This formula may also be realized directly upon noting in this case that q marks the number of parts minus one in any non-empty composition, whence

$$
F_{1}(x, y, q)=1+\frac{1}{q}\left(\frac{1-x}{1-x-x y q}-1\right)
$$

\section{Combinatorial results}

In this section, we will provide some combinatorial results concerning successions in compositions. Let $F(x, y, q)=F_{2}(x, y, q)$ denote the generating function which counts the compositions of $n$ having $d$ parts according to the number of parity successions. Taking $m=2$ in Corollary 3 gives

$$
F(x, y, q)=\frac{\left(1-x^{2}-x y(q-1)\right)\left(1-x^{2}-x^{2} y(q-1)\right)}{\left(1-x^{2}\right)^{2}-x^{3} y^{2}-x y\left(1-x^{2}\right)(1+x) q+x^{3} y^{2} q^{2}}
$$

Let $\mathcal{C}_{n, d, a}$ denote the subset of $\mathcal{C}_{n, d}$ whose members contain exactly a successions and let $c(n, d, a)=$ $\left|\mathcal{C}_{n, d, a}\right|$. Comparing coefficients of $x^{n} y^{d} q^{a}$ on both sides of (7) yields the following recurrence satisfied by the array $c(n, d, a)$. 
Theorem 5. If $n \geq 4$ and $d \geq 3$, then

$$
\begin{array}{r}
c(n, d, a)=c(n-1, d-1, a-1)+2 c(n-2, d, a)+c(n-2, d-1, a-1)+c(n-3, d-2, a) \\
-c(n-3, d-1, a-1)-c(n-3, d-2, a-2)-c(n-4, d, a)-c(n-4, d-1, a-1) .
\end{array}
$$

We can also provide a combinatorial proof of 8 , rewritten in the form

$$
\begin{aligned}
(c(n, d, a) & -c(n-2, d, a))+c(n-3, d-2, a-2)= \\
& (c(n-1, d-1, a-1)-c(n-3, d-1, a-1))+(c(n-2, d, a)-c(n-4, d, a)) \\
& +(c(n-2, d-1, a-1)-c(n-4, d-1, a-1))+c(n-3, d-2, a) .
\end{aligned}
$$

To do so, let $\mathcal{B}_{n, d, a}$ denote the subset of $\mathcal{C}_{n, d, a}$ all of whose members end in a part of size 1 or 2 . Note that for all $n, d$, and $a$, we have

$$
\left|\mathcal{B}_{n, d, a}\right|=c(n, d, a)-c(n-2, d, a),
$$

by subtraction, since $c(n-2, d, a)$ counts each member of $\mathcal{C}_{n, d, a}$ whose last part is of size 3 or more (to see this, add two to the last part of any member of $\mathcal{C}_{n-2, d, a}$, which leaves the number of parts and successions unchanged).

So to show (9), we define a bijection between the sets

$$
\mathcal{B}_{n, d, a} \cup \mathcal{C}_{n-3, d-2, a-2} \text { and } \mathcal{B}_{n-1, d-1, a-1} \cup \mathcal{B}_{n-2, d, a} \cup \mathcal{B}_{n-2, d-1, a-1} \cup \mathcal{C}_{n-3, d-2, a} .
$$

For this, we refine the sets as follows. In the subsequent definitions, $x, y$, and $z$ will denote an odd number, an even number, or a number greater than or equal three, respectively. First, let $\mathcal{B}_{n, d, a}^{(i)}, 1 \leq i \leq 4$, denote, respectively, the subsets of $\mathcal{B}_{n, d, a}$ whose members (1) end in either $1+1$ or $x+1+2$ for some $x$, (2) end in $y+2+1$ or $2+2$ for some $y$, (3) end in $x+2+1$ or $y+1+2$, or (4) end in $z+1$ or $z+2$ for some $z$. Let $\mathcal{B}_{n-1, d-1, a-1}^{(i)}, 1 \leq i \leq 3$, denote the subsets of $\mathcal{B}_{n-1, d-1, a-1}$ whose members end in $1, x+2$ for some $x$, or $y+2$ for some $y$, respectively. Finally, let $\mathcal{B}_{n-2, d-1, a-1}^{(i)}, 1 \leq i \leq 3$, denote the subsets of $\mathcal{B}_{n-2, d-1, a-1}$ whose members end in $x+1, y+1$, or 2 , respectively.

So we seek a bijection between $\left(\cup_{i=1}^{4} \mathcal{B}_{n, d, a}^{(i)}\right) \cup \mathcal{C}_{n-3, d-2, a-2}$ and

$$
\left(\cup_{i=1}^{3} \mathcal{B}_{n-1, d-1, a-1}^{(i)}\right) \cup\left(\cup_{i=1}^{3} \mathcal{B}_{n-2, d-1, a-1}^{(i)}\right) \cup \mathcal{B}_{n-2, d, a} \cup \mathcal{C}_{n-3, d-2, a} .
$$

Simple correspondences as described below show the following:

(i) $\left|\mathcal{B}_{n, d, a}^{(1)}\right|=\left|\mathcal{B}_{n-1, d-1, a-1}^{(1)} \cup \mathcal{B}_{n-1, d-1, a-1}^{(2)}\right|$,

(ii) $\left|\mathcal{B}_{n, d, a}^{(2)}\right|=\left|\mathcal{B}_{n-2, d-1, a-1}^{(2)} \cup \mathcal{B}_{n-2, d-1, a-1}^{(3)}\right|$,

(iii) $\left|\mathcal{B}_{n, d, a}^{(3)}\right|=\left|\mathcal{C}_{n-3, d-2, a}\right|$,

(iv) $\left|\mathcal{B}_{n, d, a}^{(4)}\right|=\left|\mathcal{B}_{n-2, d, a}\right|$,

(v) $\left|\mathcal{C}_{n-3, d-2, a-2}\right|=\left|\mathcal{B}_{n-2, d-1, a-1}^{(1)} \cup \mathcal{B}_{n-1, d-1, a-1}^{(3)}\right|$. 
For (i), we remove the right-most 1 within a member of $\mathcal{B}_{n, d, a}^{(1)}$, while for (ii), we remove the right-most 2 within a member of $\mathcal{B}_{n, d, a}^{(2)}$. To show (iii), we remove the final two parts of $\lambda \in \mathcal{B}_{n, d, a}^{(3)}$ to obtain the composition $\lambda^{\prime}$. Note that $\lambda^{\prime} \in \mathcal{C}_{n-3, d-2, a}$ and that the mapping $\lambda \mapsto \lambda^{\prime}$ is reversed by adding $1+2$ or $2+1$ to a member of $\mathcal{C}_{n-3, d-2, a}$, depending on whether the last part is even or odd, respectively. For (iv), we subtract two from the penultimate part of $\lambda \in \mathcal{B}_{n, d, a}^{(4)}$, which leaves the number of successions unchanged. Finally, for (v), we add either a part of size 1 or 2 to $\lambda \in \mathcal{C}_{n-3, d-1, a-2}$, depending on whether the last part of $\lambda$ is odd or even, respectively. Combining the correspondences used to show (i)-(v) yields the desired bijection and completes the proof.

We will refer to a composition having no parity successions as parity-alternating. We now wish to enumerate parity-alternating compositions having a fixed number of parts. Setting $q=0$ in (7), and expanding, gives

$$
\begin{aligned}
F & (x, y, 0)=\frac{\left(1-x^{2}+x^{2} y\right)\left(1-x^{2}+x y\right)}{\left(1-x^{2}\right)^{2}-x^{3} y^{2}}=\frac{\left(1+\frac{x^{2} y}{1-x^{2}}\right)\left(1+\frac{x y}{1-x^{2}}\right)}{1-\frac{x^{3} y^{2}}{\left(1-x^{2}\right)^{2}}} \\
& =\left(1+\frac{x^{2} y}{1-x^{2}}\right)\left(1+\frac{x y}{1-x^{2}}\right) \sum_{i \geq 0} \frac{x^{3 i} y^{2 i}}{\left(1-x^{2}\right)^{2 i}} \\
& =\sum_{i \geq 0}\left(2 y^{2 i} \sum_{j \geq 2 i-1}\left(\begin{array}{c}
j \\
2 i-1
\end{array}\right) x^{2 j-i+2}+y^{2 i+1} \sum_{j \geq 2 i}\left(\begin{array}{c}
j \\
2 i
\end{array}\right) x^{2 j-i+2}+y^{2 i+1} \sum_{j \geq 2 i}\left(\begin{array}{c}
j \\
2 i
\end{array}\right) x^{2 j-i+1}\right) .
\end{aligned}
$$

Extracting the coefficient of $x^{n} y^{m}$ in the last expression yields the following result.

Proposition 6. If $n \geq 1$ and $d \geq 0$, then

$$
c(n, 2 d, 0)= \begin{cases}2\left(\frac{n+d}{2 d-1}-1\right), & \text { if } n \equiv d(\bmod 2) \\ 0, & \text { otherwise }\end{cases}
$$

and

$$
c(n, 2 d+1,0)= \begin{cases}\left(\frac{n+d}{2}-1\right), & \text { if } n \equiv d(\bmod 2) ; \\ \left(\frac{n+d-1}{2 d}\right), & \text { otherwise }\end{cases}
$$

It is instructive to give combinatorial proofs of (10) and [11). For the first formula, suppose $\lambda \in \mathcal{C}_{n, 2 d, 0}$. Then $n$ and $d$ must have the same parity since the parts of $\lambda$ alternate between even and odd values. In this case, the number of possible $\lambda$ is twice the number of integral solutions to the equation

$$
\sum_{i=1}^{d}\left(x_{i}+y_{i}\right)=n
$$

where each $x_{i}$ is even, each $y_{i}$ is odd, and $x_{i}, y_{i}>0$. Note that the number of solutions to (12) is the same as the number of positive integral solutions to $\sum_{i=1}^{d}\left(u_{i}+v_{i}\right)=\frac{n+d}{2}$, which is $\left(\frac{n+d}{2}-1\right)$, upon letting 
$u_{i}=\frac{x_{i}}{2}$ and $v_{i}=\frac{y_{i}+1}{2}$. Thus, there are $2\left(\frac{\frac{n+d}{2}-1}{2 d-1}\right)$ members of $\mathcal{C}_{n, 2 d, 0}$ when $n$ and $d$ have the same parity, which gives (10).

On the other hand, note that members of $\mathcal{C}_{n, 2 d+1,0}$, when $n$ and $d$ have the same parity, are synonymous with positive integral solutions to

$$
\sum_{i=1}^{d}\left(x_{i}+y_{i}\right)+z=n
$$

where the $x_{i}$ are even, the $y_{i}$ are odd, and $z$ is even. Upon adding 1 to each $y_{i}$, and halving, the number of such solutions is seen to be $\left(\frac{n+d}{2}-1\right)$. Similarly, there are $\left(\frac{\frac{n+d-1}{2}}{2 d}\right)$ members of $\mathcal{C}_{n, 2 d+1,0}$ when $n$ and $d$ differ in parity, which gives (11).

Let $a(n)=\sum_{d=0}^{n} c(n, d, 0)$. Note that $a(n)$ counts all parity-alternating compositions of $n$. Taking $a=0$ in 8 and summing over $d$ yields the following result.

Proposition 7. If $n \geq 4$, then

$$
a(n)=2 a(n-2)+a(n-3)-a(n-4),
$$

with $a(0)=a(1)=a(2)=1$ and $a(3)=3$.

Summing the formulas in Proposition 6 over $d$ with $n$ fixed, and using the fact $\left(\begin{array}{l}a \\ b\end{array}\right)=\left(\begin{array}{c}a-2 \\ b\end{array}\right)+2\left(\begin{array}{c}a-2 \\ b-1\end{array}\right)+$ $\left(\begin{array}{l}a-2 \\ b-2\end{array}\right)$, yields the following pair of binomial identities, which we were unable to find in the literature.

Corollary 8. If $n \geq 0$, then

$$
a(2 n)=\sum_{d=0}^{\left\lfloor\frac{n+1}{3}\right\rfloor}\left(\begin{array}{c}
n+d+1 \\
4 d
\end{array}\right)
$$

and

$$
a(2 n+1)=\sum_{d=0}^{\left\lfloor\frac{n}{3}\right\rfloor}\left(\begin{array}{c}
n+d+2 \\
4 d+2
\end{array}\right)
$$

where $a(m)$ is given by (14).

Note that both sides of $(15)$ and $(16)$ are seen to count the parity-alternating compositions of $2 n$ and $2 n+1$, respectively, the right-hand side by the number of parts. Using (14), the binomial sums in (15) and $[16$ can be shown to satisfy fourth order recurrences; see [8] for other examples of recurrent binomial sums.

\section{Asymptotics}

We recall from (4) that $F_{m}(x, y, q)$ is a rational function. Specializing variables we obtain $F_{m}(x, 1,0)=$ $\sum_{n \geq 0} a_{n} x^{n}$, which we have seen in the previous section. The exact formulas given there for the coefficient $a_{n}$ are complemented here by asymptotic results. These are analogous to known results for Smirnov words and Carlitz compositions.

Note that $F_{m}(x, 1,0)=1 / H_{m}(x)$, where $H_{m}(x)=1-\sum_{a=1}^{m} \frac{x^{a}}{1+x^{a}-x^{m}}$. Each $1+x^{a}-x^{m}$ is analytic and so its modulus over each closed disk centered at 0 is maximized on the boundary circle. It can be 
shown that when $|x|$ is fixed, $\left|1+x^{a}-x^{m}\right|$ is maximized when $x^{a}-x^{m}$ is positive real, and minimized when $x^{a}-x^{m}$ is negative real. Furthermore, the maximum over $a$ of this maximum value occurs when $a=1$, and similarly for the minimum.

By Pringsheim's theorem, there is a minimal singularity of $F_{m}$ on the positive real axis, and this is precisely the smallest positive zero $\rho_{m}$ of $H_{m}$. Furthermore, because $F_{m}$ is not periodic, this singularity is the unique one of that modulus. Thus $F_{m}$ is analytic in the open disk centered at 0 with radius $\rho_{m}$. Note that $\rho_{m} \geq 1 / 2$ because the exponential growth rate of unrestricted compositions is 2 , and so our restricted class of compositions must grow no faster. However $\rho_{m} \leq 1$ because the sum defining $H_{m}$ has value $m$ when $x=1$. Since $\rho_{m}$ is the smallest positive solution of

$$
\sum_{a=1}^{m} \frac{\rho^{a}}{1+\rho^{a}-\rho^{m}}=1,
$$

it follows that $\rho_{m}$ is an algebraic number of degree at most $m^{2}$. Note that for all $m$ and $0<x<1$,

$$
\begin{aligned}
H_{m}(x)-H_{m+1}(x) & =x^{m+1}+\sum_{a=1}^{m}\left[\frac{x^{a}}{\left(1+x^{a}-x^{m+1}\right)}-\frac{x^{a}}{\left(1+x^{a}-x^{m}\right)}\right] \\
& =x^{m+1}+\sum_{a=1}^{m} \frac{x^{a+m}(x-1)}{\left(1+x^{a}-x^{m+1}\right)\left(1+x^{a}-x^{m}\right)} \\
& =(1-x)\left(\frac{x^{m+1}}{1-x}-\sum_{a=1}^{m} \frac{x^{a+m}}{\left(1+x^{a}-x^{m+1}\right)\left(1+x^{a}-x^{m}\right)}\right) \\
& >(1-x)\left(\frac{x^{m+1}}{1-x}-\sum_{a=1}^{m} x^{a+m}\right)>(1-x)\left(\frac{x^{m+1}}{1-x}-\sum_{a \geq 1} x^{a+m}\right)=0 .
\end{aligned}
$$

Thus $0.5 \leq \rho_{m+1}<\rho_{m} \leq \rho_{2}<0.68$ for all $m$.

The rest of the proof should proceed according to a familiar outline: apply Rouché's theorem to locate the dominant singularity of $F_{m}(x, 1,0)$, by approximating $H_{m}$ with a simpler function having a unique zero inside an appropriately chosen disk of radius $c$, where $\rho_{m}<c<1$; derive asymptotics for the coefficients $a_{n}$ via standard singularity analysis. This technique has been used in several similar problems, for example for Carlitz compositions.

There are some difficulties with this approach in our case. If we attack $F_{m}$ directly, we must derive a result for all $m$. Since $F_{m}$ is rational with numerator and denominator of degree at most $m^{2}$, for fixed $m$, we could consider using numerical root-finding methods, though for arbitrary symbolic $m$ this will not work. It is intuitively clear that for sufficiently large $m, F_{m}$ should be close to $F_{\infty}$ and so by using Rouché's theorem, we could reduce to the Carlitz case.

However, even the Carlitz case is not as easy as claimed in the literature, and we found several unconvincing published arguments. Some authors simply assert that $F_{\infty}$ has a single root, based on a graph of the function on a given circle. This can be made into a proof, by approximately evaluating $F_{\infty}$ at sufficiently many points and using an upper bound on the best Lipschitz constant for the function, but this is somewhat unpleasant. We do not know a way of avoiding this problem - the minimum modulus of a function on a circle in the complex plane must be computed somehow. We use an approach similar to that taken in [9]. 
The obvious approximating function to use is an initial segment with $k$ terms of the partial sum defining $F_{m}$. However, it seems easier to use the initial segment of the sum defining $F_{\infty}$, which we denote by $S_{k}$. We will take $k=7$ and $c=0.7$ and denote $S_{7}$ by $h$. By using the Jenkins-Traub algorithm as implemented in the Sage command maxima.allroots (), we see that all roots of $h$, except the real positive root (approximately 0.572 ) have real or imaginary part with modulus more than 0.7 , so they certainly lie outside the circle $C$ given by $|x|=c$.

To apply Rouché's theorem, we need an upper bound for $\left|H_{m}-h\right|$ on $C$ which is less than the lower bound for $|h|$ on $C$. We first claim that the lower bound for $|h|$ on $C$ is at least 0.43 . This can be proved by evaluation at sufficiently many points of $C$. Since $\left|h^{\prime}(z)\right|$ is bounded by 100 on $C, N:=1000$ points certainly suffice. This is because the minimum of $|h|$ at points of the form $0.7 \exp (2 \pi i j / N)$, for $0 \leq j \leq N$, is more than 0.51 (computed using Sage), and the distance between two such points is at most $8 \times 10^{-4}$, by Taylor approximation. In fact, it seems that the minimum indeed occurs at $x=0.7$, but this is not obvious to us.

We now compute an upper bound for $\left|H_{m}-h\right|$. To this end, we compute, when $m \geq 7$,

$$
\begin{aligned}
\left|H_{m}(x)-h(x)\right| & =\sum_{a=8}^{m} \frac{x^{a}}{1-x^{a}+x^{m}}+\left(\sum_{a=1}^{7} \frac{x^{a}}{1-x^{a}+x^{m}}-h(x)\right) \\
& \leq \sum_{a=8}^{\infty} \frac{c^{a}}{1-c^{8}}+\left(\sum_{a=1}^{7} \frac{x^{a}}{1-x^{a}+x^{m}}-h(x)\right) .
\end{aligned}
$$

The sum $\sum_{a=8}^{\infty} \frac{c^{a}}{1-c^{8}}$ has value less than 0.204 when $c=0.7$. The second sum is smaller than 0.2 which can be verified by a similar argument to the above, by evaluating at sufficiently many points.

We still need to deal with the cases $m<7$ and these can be done directly via inspection after computing all roots numerically as above.

The above arguments show that $\rho_{m}$ is a simple zero of $H_{m}$ and hence a simple pole of the rational function $F_{m}(x, 1,0)$. The asymptotics now follow in the standard manner by a residue computation, and we obtain

$$
a_{n} \sim \rho_{m}^{-n} \frac{1}{-\rho_{m} H^{\prime}\left(\rho_{m}\right)}
$$

For example, $F_{2}(x, 1,0)=\left(1+x-x^{2}\right) /\left(\left(1-x^{2}\right)^{2}-x^{3}\right)$ has a minimal singularity at $\rho_{2} \approx$ 0.6710436067037893 , which yields the following result.

Theorem 9. We have

$$
a(n) \sim(0.6436) 1.4902^{n}
$$

for large $n$, where $a(n)$ is given by (14).

For example, when $n=20$, the relative error in this approximation is already less than $0.2 \%$. The exponential rate $1 / \rho_{m}$ approaches the rate for Carlitz compositions, namely $1.750 \cdots$, as $m \rightarrow \infty$.

For a given $m$, it is possible in principle to compute asymptotics in a given direction by analysis of $F_{m}(x, y, q)$, for example, using the techniques of Pemantle and Wilson [17]. We provide here a sketch for the case $m=2$, the proof being similar for other small $m$, and refer the reader to the above reference 
or the more recent book [18]. In this case we have

$$
\begin{aligned}
F_{2}(x, y, q) & =\left(1-\frac{x y}{1-x^{2}-x y(q-1)}+\frac{x^{2} y}{1-x^{2}-x^{2} y(q-1)}\right)^{-1} \\
& =\frac{\left(1-x^{2}-x y(q-1)\right)\left(1-x^{2}-x^{2} y(q-1)\right)}{1-2 x^{2}-q x y+x^{4}-q x^{2} y-x^{3} y^{2}+q x^{3} y+q x^{4} y+q^{2} x^{3} y^{2}} .
\end{aligned}
$$

By standard algorithms, for example as implemented in Sage's solve command, one can check that the partial derivatives $H_{x}, H_{y}, H_{q}$ never vanish simultaneously, so that the variety defined by $H_{m}$ is smooth everywhere. The critical point equations are readily solved by the same method. For example, for the special case when $n=2 d=4 t$, where $t$ denotes the number of congruence successions, we obtain (using the Sage package amgf [19]) the first order asymptotic

$$
(0.379867842273)(15.8273658508862)^{t} /(\pi t),
$$

which has relative error just over $1 \%$ when $n=32$ (the number of such compositions being 54865800 ). Bivariate asymptotics when $q=0$, or when $y=1$, could be derived similarly. The smoothness of the variety defined by $H_{m}$ leads quickly to Gaussian limit laws in a standard way as described in [18], and we leave the reader to explore this further.

\section{References}

[1] M. Abramson and W. Moser, Generalizations of Terquem's problem, J. Combin. Theory 7 (1969) $171-180$.

[2] E. A. Bender and E. R. Canfield, Locally restricted compositions I. restricted adjacent differences, Electron. J. Combin. 12 (2005) \#R57.

[3] E. A. Bender and E. R. Canfield, Locally restricted compositions II. general restrictions and infinite matrices, Electron. J. Combin. 16 (2009) \#R108.

[4] E. A. Bender and E. R. Canfield, Locally restricted compositions III. adjacent-part periodic inequalities, Electron. J. Combin. 17 (2010) \#R145.

[5] E. A. Bender, E. R. Canfield, and Z. Gao, Locally restricted compositions IV. nearly free large parts and gap-freeness 19(4) (2012) \#P14.

[6] L. Carlitz, Restricted compositions, Fibonacci Quart. 14 (1976) 254-264.

[7] P. Chinn and S. Heubach, Compositions of $n$ with no occurrence of $k$, Congr. Numer. 164 (2003) 33-51.

[8] C. Elsner, On recurrence formulae for sums involving binomial coefficients, Fibonacci Quart. 43 (2005) 31-45.

[9] W. M. Y. Goh and P. Hitczenko, Average number of distinct part sizes in a random Carlitz composition, European J. Combin. 23 (2002) 647-657. 
[10] S. Heubach and S. Kitaev, Avoiding substrings in compositions, Congr. Numer. 202 (2010) 87-95.

[11] S. Heubach and T. Mansour, Combinatorics of Compositions and Words, CRC Press, Boca Raton, 2010.

[12] A. Knopfmacher, A. O. Munagi, and S. Wagner, Successions in words and compositions, Ann. Comb. 16 (2012) 277-287.

[13] T. Mansour and A. O. Munagi, Alternating subsets modulo m, Rocky Mountain J. Math. 42 (2012) 1313-1325.

[14] A. O. Munagi, Alternating subsets and permutations, Rocky Mountain J. Math. 40 (2010) 19651977.

[15] A. O. Munagi, Alternating subsets and successions, Ars Combin. 110 (2013) 77-86.

[16] A. O. Munagi, Parity-alternating permutations and successions, Cent. Eur. J. Math. 12 (2014) 13901402 .

[17] R. Pemantle and M. C. Wilson, Asymptotics of multivariate sequences I: smooth points, J. Combin. Theory Ser. A 97 (2002) 129-161.

[18] R. Pemantle and M. C. Wilson, Analytic Combinatorics in Several Variables, Cambridge University Press, 2013.

[19] A. Raichev, Sage package amgf, available from https://github.com/araichev/amgf. Accessed 2013-07-10.

[20] S. Tanimoto, Parity-alternate permutations and signed Eulerian numbers, Ann. Comb. 14 (2010) $355-366$.

[21] S. M. Tanny, Permutations and successions, J. Combin. Theory Ser. A 13 (1975) 55-65.

[22] S. M. Tanny, On alternating subsets of integers, Fibonacci Quart. 13 (1975) 325-328. 